\title{
Can a Free Wearable Activity Tracker Change Behavior? The Impact of Trackers on Adults in a Physician-Led Wellness Group
}

Lisa Gualtieri ${ }^{1}$, PhD, ScM; Sandra Rosenbluth ${ }^{1}$, MS; Jeffrey Phillips ${ }^{2}$, MD

${ }^{1}$ Department of Public Health and Community Medicine, Tufts University School of Medicine, Boston, MA, United States

${ }^{2}$ Family Doctors, LLC, Swampscott, MA, United States

Corresponding Author:

Lisa Gualtieri, $\mathrm{PhD}, \mathrm{ScM}$

Department of Public Health and Community Medicine

Tufts University School of Medicine

136 Harrison Avenue

Boston, MA, 02111

United States

Phone: 16176360438

Fax: 16176364017

Email: lisa.gualtieri@tufts.edu

\begin{abstract}
Background: Wearable activity trackers (trackers) are increasingly popular devices used to track step count and other health indicators. Trackers have the potential to benefit those in need of increased physical activity, such as adults who are older and who face significant health challenges. These populations are least likely to purchase trackers and most likely to face challenges in using them, yet may derive educational, motivational, and health benefits from their use once these barriers are removed.
\end{abstract}

Objective: The aim of this research was to investigate the use of trackers by older adults with chronic medical conditions who had never used trackers previously. Our primary research questions were (1) if participants would accept and use trackers to increase their physical activity; (2) if there were barriers to use besides cost and training; (3) if trackers would educate participants on their baseline and ongoing activity levels and support behavior change; and (4) if clinical outcomes would show improvements in participants' health.

Methods: This study was conducted with 10 patients in a 12 week physician-led wellness group offered by Family Doctors, LLC. Patients were given trackers in the second week of the wellness group and were interviewed 2-4 weeks after it ended. The study investigators analyzed the interview notes to extract themes about the participants' attitudes and behavior changes and collected and analyzed participants' clinical data, including weight and LDL-Cholesterol (LDL), over the course of the study.

Results: Over the 12-14 weeks of tracker use, improvements were seen in clinical outcomes, attitudes towards the trackers, and physical activity behaviors. Participants lost an average of a half-pound per week ( $\mathrm{SD}=0.408)$, with a mean total weight loss of 5.97 pounds $(P=.0038)$. Other short-term clinical outcomes included a 9.2\% decrease in LDL levels $(P=.0377)$. All participants reported an increase in well-being and confidence in their ability to lead more active lives. We identified 6 major attitudinal themes from our qualitative analysis of the interview notes: (1) barriers to tracker purchase included cost, perceived value, and choice confusion; (2) attitudes towards the trackers shifted for many, from half of the participants expressing excitement and hope and half expressing hesitation or trepidation, to all participants feeling positive towards their tracker at the time of the interviews; (3) trackers served as educational tools for baseline activity levels; (4) trackers provided concrete feedback on physical activity, which motivated behavior change; (5) tracker use reinforced wellness group activities and goals; and (6) although commitment to tracker use did not waver, external circumstances influenced some participants' ongoing use.

Conclusions: Our findings suggest that adding trackers to wellness groups comprising older adults with chronic medical conditions can support education and behavior change to be more physically active. The trackers increased participant self-efficacy by providing a tangible, visible reminder of a commitment to increasing activity and immediate feedback on step count and progress towards a daily step goal. While acceptance was high and attitudes ultimately positive, training and support are needed and short-term drop-off in participant use is to be expected. Future research will further consider the potential of trackers in older adults with chronic medical conditions who are unlikely to purchase them, and studies will use larger samples, continue over a longer period of time, and evaluate outcomes independent of a wellness group. 
(iproc 2016;2(1):e1) doi: 10.2196/iproc.6245

\section{KEYWORDS}

wearable activity trackers; fitness trackers; trackers; physical activity; chronic disease; behavior change; wellness group; wellness; older adults; digital health

This poster was presented at the Connected Health Symposium 2016, October 20-21, Boston, MA, United States. The poster is displayed as an image in Figure 1 and as a PDF in Multimedia Appendix 1.

Figure 1. Poster.

\section{Can a free wearable activity tracker change behavior? The impact of trackers on adults in a physician-led wellness group Lisa Gualtieri, PhD, ScM'1 , Sandra Rosenbluth, MS'1, Jeffrey Phillips, MD ${ }^{1,2}$ \\ 1. Tufts University School of Medicine, 2. Family Doctors, LLC}

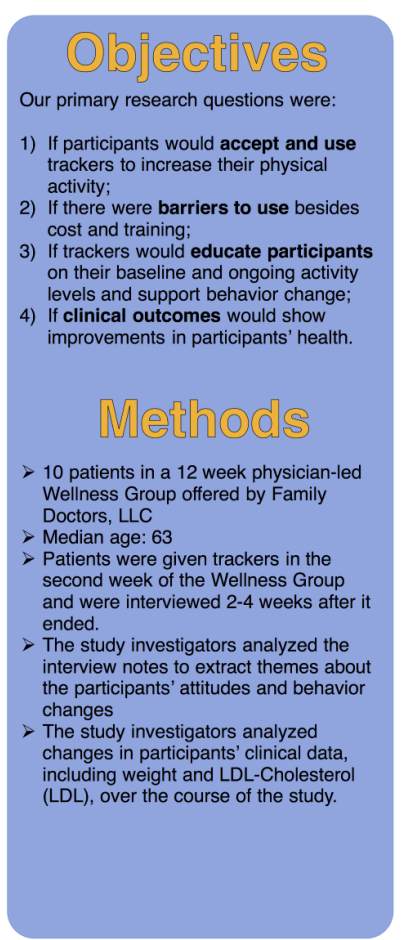

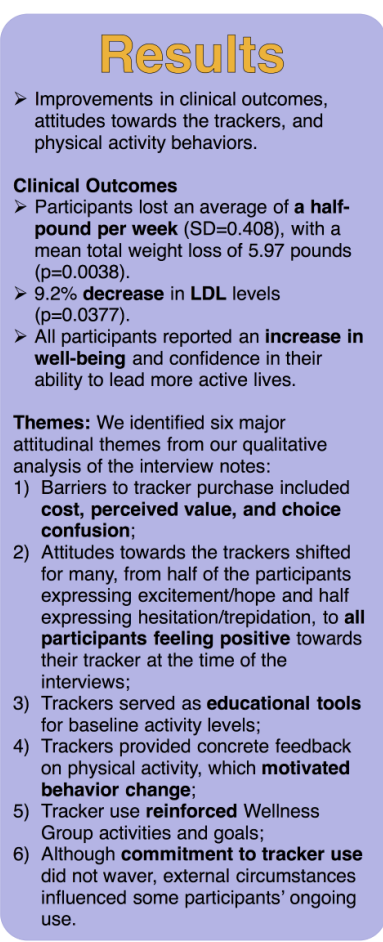
use.

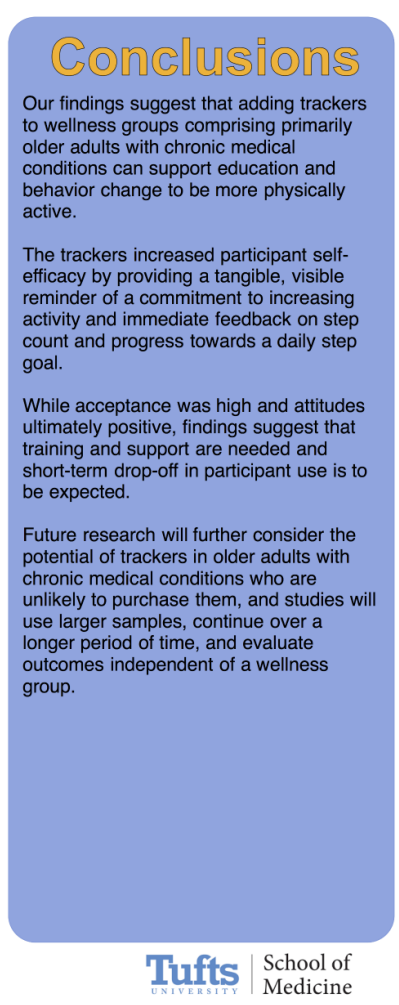

\section{Multimedia Appendix 1}

Poster.

[PDF File (Adobe PDF File), 306KB-Multimedia Appendix 1]

Edited by T Hale; submitted 20.06.16; peer-reviewed by CHS Scientific Program Committee; accepted 02.08.16; published 15.12.16

\section{Please cite as:}

Gualtieri L, Rosenbluth S, Phillips J

Can a Free Wearable Activity Tracker Change Behavior? The Impact of Trackers on Adults in a Physician-Led Wellness Group iproc 2016;2(1):e1

URL: http://www.iproc.org/2016/1/e1/

doi: 10.2196/iproc.6245

PMID: 
CLisa Gualtieri, Sandra Rosenbluth, Jeffrey Phillips. Originally published in Iproceedings (http://www.iproc.org), 15.12.2016. This is an open-access article distributed under the terms of the Creative Commons Attribution License (http://creativecommons.org/licenses/by/2.0/), which permits unrestricted use, distribution, and reproduction in any medium, provided the original work, first published in Iproceedings, is properly cited. The complete bibliographic information, a link to the original publication on http://www.iproc.org/, as well as this copyright and license information must be included. 